\title{
Data Collection and New Technology
}

\author{
http://dx.doi.org/10.3991/ijet.v8i2.2157 \\ Olubunmi. Philip Aborisade, Ph.D. \\ College of New Rochelle, New York, USA
}

\begin{abstract}
Interview has become a popular method of data collection in qualitative research. This article examines the different interview methods for collecting data (e.g., structured interviews, group interviews, unstructured, etc.), as well as the various methods for analyzing interview data (e.g., interpretivism, social anthropology, collaborative social research). It also evaluates the interview types and analysis methods in qualitative research and the new technology for conducting interviews such as e-mail, telephone, skype, webcam, Facebook chat etc to ascertain how they limit interviewees from giving full picture of moral and ethical Issues.
\end{abstract}

Index Terms-Ethical and moral issues, Methodology, Qualitative research, Technology assisted Interview.

\section{INTRODUCTION}

Two major research methods stand out in research tradition-qualitative and quantitative (Williams, 1992). Researchers use quantitative research when there is need for measurement of phenomenon, hypothesis testing, and generalization. Qualitative research is utilized when there is need for researchers to work with words and images. The strength of qualitative research lies in the use of interviews which allow qualitative researchers to conduct an in-depth investigation into their subject of inquiry. Charles Booth first used interview in a social survey when he conducted a survey of the economic and social conditions in London in 1886 (Converse, 1987 cited in Fontana and Frey, 2000).

Over the years, interviews have emerged the primary technique for data collection in qualitative methodologies (Cooper and Schindler, 2003). The five acceptable approaches in qualitative methods-ethnography, case study, grounded theory, phenomenology, and heuristic or heuristic phenomenology (Creswell, 2007) therefore rely on different types of interviews for data collection.

The type of interview employed for data collection in qualitative research is determined by the number of people involved in the interview, the level of structure, proximity of interviewer to participants, and the number of interviews conducted during the research (Cooper and Schindler, 2003). Against the background of these factors, contemporary literature identifies different types of interviews such as individual and group interview.

Additionally, qualitative researchers have at their disposal, unstructured interview (with close-ended questions) with no specific questions or order of topics to be discussed, semi- structured interview with few specific questions and follow-up questions, and structured interview which requires questionnaire. Questions asked in this regard are mostly open-ended (Cooper and Schindler, 2003).
Besides interview, other forms of data collection continue to emerge in qualitative methods. These include observation (participant or nonparticipant), document (private or public), audiovisual materials (photographs, compact disks, and videotapes), journaling in narrative storytelling, and e-mail text messages, (Creswell, 2007). With the emergent of social media, interviews are being conducted using media modalities like facebook, twitter and MySpace for data collection in qualitative research. The question therefore is whether the use of these technological tools will limit interviewees from giving full picture of moral and ethical Issues.

Given the predominant roles of interview in qualitative research, it has become imperative for researchers to acquire interviewing skills-including the use of social media. These skills according to Cooper and Schindler (2003) include "making respondents comfortable, probing for details without making the respondents feel harassed, remaining neutral while encouraging the participant to talk openly, listening carefully, following participants train of thought, and extracting insights from hours of detailed descriptive dialogue” (p. 170).

\section{Qualitative Methodologies FOR COLLECTING DATA}

There are different data collection methodologies available to the qualitative researcher. As data can take different forms and shapes, there is no single type of data collection methodology. However, data can take the form of field notes, interview transcript, transcribed recordings of naturally occurring interaction, documents, pictures, and other graphic representations. (Coffey and Atkinson, 1996). Tesch (1990 cited in Coffey and Atkinson), identifies about 26 analytic strategies for data collection available to the qualitative researcher.

As qualitative researchers continue to utilize the abovementioned forms of data collection tools, their differences and similarities continue to emerge in literature. These differences and similarities can therefore be viewed from different perspectives. It could be in terms of research objective, topic, or participants.

In comparing and contrasting individual interviews with group interviews, it is suffice to say that, individual interviews and group interviews are similar in the sense that, they are both qualitative data collection methods, but differ in terms of what they set out to achieve. As Cooper and Schindler (2003) argue, individual interviews explore the life of individuals, creates case history through repeated interviews, and tests surveys. Group interviews get the researchers accustom to a field and language of inquiry, explore a range of attitudes, opinions, behaviors, observe a process of consensus and disagreement, and add contextual detail to the outcome of qualitative research. 
Another comparison of individual and group interviews could be drawn from topic concerns which allow individual interviews to carry out detailed individuals' experience, choices, and biographies and explore sensitive issues that might cause anxiety. Group interviews are concerned with issues of public interest or common concern where little is known (Cooper and Schindler, 2003).

From the participants' point of view, it could be argued that individual interviews accommodate time-pressed participants such as elite who may choose a convenient time and place for the interview, those with language skills to articulate their views and participants with distinctions that may inhibit participation in group interviews. The situation differs in group interviews because participants have similar backgrounds, they can articulate their views and offer a range positions on issues (Cooper and Schindler, 2003).

The two types of interviews cater for two types of participants so as to be able to gather needed qualitative data more efficiently and effectively. However, the fact remains that, both individual and group interviews are qualitative data collection methods designed to extract information from participants either individually or in a group.

Stockes and Bergin (2006) introduce an interesting dimension to the use of interviews for data collection in qualitative research. They argue that, group interviews, have "extrinsic" advantages such as speed and cost while individual interviews have "intrinsic" advantages in terms of quality of outcome. Using a parallel study of a single business issue, the authors argue that, group interviews have considerable influence on the consensus views expressed in focus groups not representative of respondents' individual groups. "The groups were unable to match the depth and detail generated by individual interviews and to uncover subtleties in attitudes. The interviews offer less breadth of data and contextual information” (Stockes and Bergin, 2006).

While focus group interviews may be less expensive and faster in terms of data collection, Stockes and Bergin (2006) argue that, individual interviews demonstrate superiority over group interviews in uncovering important underlining issues. In terms of originality and value, the authors' parallel research confirms "focus group interviews do not justify their predominance as a market research method in preference to individual interviews on the grounds of qualitative outcomes alone" (Stockes and Bergin, 2006, p. 26).

Structured, semi structured and unstructured interviews differ structurally. While unstructured interviews generally start with specific questions or order of topic with open-ended questions, structured interviews often use detailed interviews akin to questionnaire with closedended questions, semi structure interviews start with few questions juxtaposed with follow up questions (Cooper and Schindler, 2003).

It is arguable that, the amount of information gathered through semi structured interviews depends on researchers' ability to ask probing questions. However, in structured interview, the amount of information to be gathered will depend on the type of questions asked. With unstructured interviews, there is a very high possibility that participants do most of the talking since there is no specific question or order of topic. Questions asked in this regard are close-ended. This type of data collection method does not allow participants to go beyond the scope of questions asked. This type of method may or may not generate needed data, but it is a good way to gather data for qualitative studies.

\section{E-MAIL, TELEPHONE AND AUDIOVISUAL INTERVIEWS}

With technology, e-mail interviewing and the use of audiovisual technologies become veritable tools in qualitative research. While e-mail interviewing provides a viable alternative to face-to-face interviews, audiovisual interview with modern media tools like skype, facebook and webcam are becoming more popular. This is because they appeal to people's sense of hearing and vision. These make them more effective and pave way for better understanding of data. There is also the Computer Assisted Telephone interview which allows interviewers to ask questions over the telephone and key the answer into the computer system immediately by the interviewer. Also the use of private chats equally provides interview opportunities without meeting face-to-face.

Additionally, e-mail is widely acclaimed as having the capacity to reach "participants of large or geographically dispersed sample of people by sending them e-mail message boards or discussion groups rather than making long distance telephone calls, using regular mail or travelling to the location of participants” (Meho, 2006, p. 1284). By using e-mail for data collection, Meho (2006) argues that, "researchers save the cost of transcribing recorded interviews and time that could have been spent editing transcribed texts. This is because e-mail is in electronic format and electronic format needs little editing or formatting before they are processed for analysis” (p. 1285). However, critics of audiovisual materials in qualitative research have argued that, it is expensive, time consuming, and needs professional training to handle. Thus, most researchers are not always disposed to this type of data collection tool as they do to interviews.

Unlike audiovisual material, e-mail is less expensive and can reach as many participants as possible (Meho, 2006). Participants in an e-mail can remain anonymous and respond to the e-mail at their convenient time, this may not be possible in case of audiovisual materials. In addition, e-mail can be used to reach participants that are very difficult to reach. However, e-mail needs some level of literacy which makes it difficult for those who are not computer literate or who cannot read or write fit into any research using interviews by e-mail. Giving the peculiar nature of participants in an e-mail interview, it is arguable that e-mail generates low and incomplete responses, and requires follow ups that may not generate sufficient number of responses (Meho, 2006).

Despite the fact that e-mail limits the researcher to participants with access to the Internet, it "democratizes and internationalizes research” (Meho, 2006, p. 1284). But in sharp contrast to face-to-face, or telephone interviewing, e-mail has the capability to enable researchers study individuals or groups with special characteristics or those that are very difficult to reach or interview by phone.

According to Kim Hart (2006, Dec./Jan.), “e-mail interview has become an increasingly popular technique. It eliminates endless rounds of phone tag, and it gives sources a chance to provide well-thought-out answers 
rather than top-of-the-head responses. But critics warn that it's hardly a substitute for real-time conversation and may be a recipe for sterile journalism."

With e-mail, the researcher is able to interview people who are shy from face-to-face, chief executives and politicians who are always indisposed. The researcher is also able to reach out to "those who cannot express themselves as well in talking as they do in writing, especially when the language used in communication with the participants is their second one" (Karchmer, 2003; Kim et al., 2003 cited in Meho, 2006, p. 1286). It could be said therefore that, e-mail allows the researcher to study groups or communities that would not have be studied (Meho, 2006).

\section{METHODS FOR ANALYZING INTERVIEW DATA}

The same way there is no single methodology for data collection in qualitative research, there is also no single right way to data analysis. What is evident is that, analyzing interview data is cumbersome, hectic, complex, and time consuming. To analyze the different types of data the researcher is confronted with in qualitative research, three major approaches or methods are available to researchers. These are: interpretivism, social anthropology, and collaborative social research.

The three methods of analyzing interview data can also be viewed in terms of their history of origin. Interpretivism has a long intellectual history dated back to "Dilthey (19/11/1977) thesis that human discourse and action could not be analyzed with the methods of natural and physical science" (Miles and Huberman, 1994, p. 8). The argument in this thesis centers on how text (seen as human activities) is analyzed. Dilthey (1977 cited in Miles and Huberman, 1994) and phenomenologist's argument is that, deep understanding, and empathy with the subject of research is imperative and for researchers to interpret interview data accurately. For social integrationists, the answer lies in understanding of group actions and interactions while interpretation of meaning is evident in both cases (Miles and Huberman, 1994).

Social anthropology differs in the sense that it is predominantly for researchers who are concerned with behavioral regularities such as language use, artifacts, rituals relationships (often expressed as patterns or language or rules) to provide inferential key to the culture of society under study (Miles and Huberman, 1994). The major task here is to "uncover and explicate the ways in which people in a particular work setting come to understand, account for, take action and otherwise manage their day- to -day situation based on successive observation and interviews reviewed to guide the next move in the field (Van Mannen, 1979 cited in Miles and Huberman, 1994).

Collaborative social research sharply differs from both interpretative and social anthropology approaches because it is design for social action in a social setting. This is an action research to effect change. It is dated back to 1920s (Whyte, 1991 cited in Miles and Huberman, 1994).

Fontana and Frey (2000) explain why researchers using the interpretative method to analyze data may not be "neutral, unbiased and invisible" while analyzing interview data. The authors' argument is that, the enormity of the data the researcher is confronted with does not allow the researcher to be neutral. "No matter how energized the researcher may be, he or she is buried under an increasing mountain of field notes, transcripts, newspaper clippings and audio tapes” (Fontana and Frey, 2000, p. 710).

\section{TYPES OF INTERVIEW AND METHODS OF ANALYSIS}

Ethical leadership and business performance are very synonymous. The synonym continues to generate tension between performance and ethical leadership. Contemporary literature on ethical leadership places emphases on the roles of moral and ethical values within today organizations. The success or failures of today's organizations are linked to moral and ethical standards of leadership. Examples of these are evident in the numerous United States companies that continue to declare bankruptcy due to bad moral and ethical judgments of their leaders. As Victor and Cullen (1987 cited in Driscoll and Mckee, 2006) argue, "ethical organizational culture refers to shared perceptions of ethically acceptable behavior and the ways in which ethically acceptable behavior and the ways in which ethical issues are addressed in organizations” (p. 208).

The same way leaders of failed organizations receive condemnation for their moral and ethical leadership; leaders of successful organizations continue to receive kudos for making good moral and ethical judgments. From the foregoing, it is evident that success of leaders in today organizations has some connections to moral and ethical judgments. Koestenbaum, (2002) vividly captures the impacts of moral and ethical values in organizations and their leaders when he calls for a Nobel Prize for organizations that demonstrate leadership effectiveness as a result of ethical moral behavior.

In response to the roles of moral and ethical values in leader's success in organizations, leaders' morals, and ethical issues continue to receive tremendous attention in the literature (Brien, 1998; Dickson et al., 2001; Fisher and Flower, 1995; Key, 1999; Milton-Smith, 1997; Sims, 2000 cited in Driscoll and Mckee, 2006). Researchers on leadership issues have come up with appropriate types of research and analysis methods for researching leadership qualities that relate to the moral and ethical leader's success within organizations. In qualitative research, different types of interviews and analysis methods have been adjudged as appropriate for research leadership in relation to moral and ethical values.

The different types of interviews at the disposal of the qualitative researchers can be categorized into three-informal, guided and standardized (Patton, 1990). This categorization is based on the different elements that distinguish the interview types. These elements include research objective, topic of concerns, number, type of participants, and structure of the interviews (Cooper and Schindler, 2003).

\section{EVALUATION OF INTERVIEW TYPES}

\section{A. Informal interviews}

This type of interview allows the researcher to improvise questions that suits the priorities and personality of the respondent. Naturally, the interview in this regard is individualized (to gather in-depth information on one- toone basis) and unstructured (without specific questions or order of topic) and it is designed to probe unanticipated responses from respondents. This requires researchers possess interviewing skills and good knowledge of the 
subject of the interview to be able to pose probing questions that will elicit unanticipated responses from the respondents. Informal interviews pose a lot of challenges to the researcher when it comes to data analysis. This is because researchers conducting informal interviews gather avalanche of information that may be difficult to analyze or compare (Patton, 1990).

\section{B. Guided Interview}

This type of interview allows research to draw up a list of general questions, which are juxtaposed with follow up questions during interviews. Like informal interviews, the questions in guided interview are adapted to the personality and priorities of participants in the interview. As the name implies, the interview is guided by rules, but still open ended because it allows for elaborate questions and responses and not questions that demands yes or no response. Also, the researcher needs some interviewing skills to elicit unplanned responses from the participants just as in informal interviews. The beauty of a guided interview lies in its ability for discoveries of new themes and efficient data analysis (Patton, 1990).

\section{Standardized Interview}

This type of interview allows researchers to ask the same type of questions in the same order from respondents which give room for comparison of responses from participants. The questions can be open or close ended which makes the interview and data analysis efficient. Critics of this type of interview have argued that, it prevents exploration of unplanned topics and participants' concerns during interview. This may however be perfect for researchers with limited financial resources (Patton, 1990).

\section{Evaluation of Data ANAlysis Methods}

After researchers have used the appropriate types of interviews for data collection in researching leadership qualities in relation to moral and ethical values, the next task for the researcher is data analysis; to turn raw data from interviews or other data collection tools such as observation or survey, into well packaged piece of qualitative research. The method of analysis depends on the theoretical and methodological background (Lambretcht, 1999). In analyzing data for leadership qualities, interpretive method will be a perfect match. This will enable the researcher to illuminate the managerial significance of leaders in terms of moral and ethical values a leader has brought to his or her organization.

As Muldoon and Miller (2005) argue, "Managers' characteristics are found to form thematic distinctions corresponding with outcomes of leader's effectiveness and individual success” (p. 132). The use of interpretative methodology for analyzing leadership moral and ethical values will help make meaning out of in-depth interviews and other types of data collection methods. Critics of this method of analysis argue that, "the researcher is no more detached from their objects of study than their informant" (Miles and Huberman, 1994, p. 8). They added that, researchers have their understanding, convictions, conceptual orientations, and culture which are bound to influence their judgments. "If the researcher uses few preestablished instruments, it will be difficult to separate out 'external' information from what they themselves have contributed when encoding and decoding the words of their informants" (Miles and Huberman, 1994, p. 8).

The use of social ethnography method of analysis in researching leader's success in terms of moral and ethical values equally helps researchers to establish the day- today behavior of leaders in organizational setting, language of communication, and the general work ethics of the leader in organizations. By so doing, researchers uncover the way leaders understand, account for, take action, and manage their activities. The uncovering of the modes of life and operation of leaders in the organizational setting is based on data collected from observations and interviews using ethnographic method (Miles and Huberman, 1994).

Collaborative social research is also a veritable tool for researching leader's success in relation to morals and ethical values in organizations. This method is geared towards social change. In organizations with a leader that is embattled with moral and ethical issues, this method of analysis will help effect the needed change for leadership success. To effect this change, researchers first outline field experiment- changing the bad moral and ethical behavior in organizations. Researchers then collect data and give them to 'activist' as feedback to come up with the next line of action (Miles and Huberman, 1994). "This approach incorporates some of the features of naturalistic studies: participant observation, sensitivity to participants' concerns, focus on descriptive data in the initial phases, no standardized instrumentation, a holistic perspective, the search for underlying themes and pattern" (Miles and Huberman, 1994).

\section{GIVING FUll PICTURE TO INTERVIEWER}

It is a herculean task to extract information from leaders in relation the story of their success or failure. Leaders may be unwilling to tell the story of the secret behind their success for fear of emulations by competitor or reprisal by management that may prefer to keep such information secret. Leader in this type of situation may not want to give the full picture to the interviewer.

Also in the same vein, leaders with deficient moral and ethical values may not be willing to reveal such deficiencies to researchers for fear of losing their jobs should they do so. Leaders may also be unwilling to take responsibility for their bad moral and ethical values that are responsible for the failure of their leadership in their organization to save their face. These are some of the limitations faced by researchers researching leaders' success in relation to moral and ethical values in organizations.

To overcome these limitations, research can assure the interviewee that all information provided during the interview will not be attributed to the interviewee or its organization. By so doing, the leaders with concerns that may limit them from giving the full picture to the interviewer will have the assurance that giving out such information will not hurt their person or organization.

Researchers also need to seek permission of leaders being investigated and their organizations through informed consent which will give full details of the interview and purpose of the research. By so doing, interviewees will be assured that giving full account of their stories will not in any way harm them or their organizations as a result of the information divulged about their leadership and organizations. 


\section{SHORT PAPER \\ DATA Collection AND NEW TECHNOLOGY}

More importantly, the researcher can agree to let the interviewee see a draft of the interview before it is used for any purpose. With this type of agreement, the confidentiality of leaders participating in an interview can be guaranteed. This will relieve the leaders of any concern that may prevent him from giving full picture to the interviewer.

Miles and Huberman (1994) capture the need for participants and researchers to reach some 'explicit agreement' about shared expectations. This type of expectation will help researchers overcome limitations that may prevent interviewees from giving full picture of information requested by researchers. Miles and Huberman (1994) further argue that, the agreement among other things will dwell on time and effort involved in the interview, types of data to be collected, if participation voluntary, confidentiality of materials from participants, anonymity of participants, who produces descriptive and explanatory products, review and critique of interim and final product by participants, and benefit accruable to the participants.

By addressing these issues in the agreement, researchers would have secured participants' confidence in the interview process. This will help the interviewees overcome concerns that may prevent them from giving the full picture of their stories. This type of agreement is very significant in researching leaders' success in relations to moral and ethical issues in today's organizations.

The way researchers conduct interviews while researching leadership qualities can also help overcome concerns that may prevent interviewees from giving full picture. The interviewer "should be neither too grim nor too effusive; neither too talkative nor too timid. The idea should be to put the respondent at ease, so that he will talk freely and fully" (Selltiz, Jahoda, Deutsch, and Cook, 1965, p. 576, cited in Fontana and Frey, 2000, p. 170). The argument here is that, the interviewee will be will to provide full account of their story if the interview atmosphere is comfortable. It can be argued however, that, irrespective of the level of comfort, some leaders may still be unwilling to give full picture if they know doing so will hurt them or their organizations. This may not be the case when researching other issues where interviewer may be used to gain interviewees trust by providing an interview atmosphere where the interview can talk freely and fully.

\section{CONCLUSION}

In the face of continued opposition to qualitative methods by its critics as a subjective method of inquiry, it continues to stand out as a method of inquiry that goes beyond measurement of figures, but goes to deep down to the root of the subject of investigation. The depth of investigation that is credited to qualitative methods has attracted most quantitative researchers to qualitative methods as the only way to get to the root of their subject of inquiry. As Antonakis et al., (2003) argue, “ Leadership researchers typically have used quantitative approaches; however, to better understand complex, embedded phenomena, qualitative approaches to studying leadership are also necessary” (Alvesson, 1996; Bryman, Stephens, and Campo, 1996; Conger, 1998 cited in Antonakis, et al., 2003, p. 54).
The confidence that researchers repose in qualitative method is liked to interview which has over the years emerged as a veritable method of inquiry that allows indepth investigation in research. Swanson and Holton (2005) illuminate the significance of interview as a veritable tool in qualitative research when they argue that, "qualitative methods are better suited for collecting, analyzing, and interpreting respondent constructions than are quantitative methods, because they are immediate, processual, elaborative and amenable to inter-subjective interpretation” (p. 231).

\section{REFERENCES}

[1] Antonakis, J., Schriesheim, C. A., Donovan, J. A., GalakrishnaPillai, K., Pellegrini, E. K., \& Rossomme, J. L. (2003). Methods for studying leadership. Retrieved January 20, 2009 from http://www.hec.unil.ch/jantonakis/Antonakis\%20Chapter\%2003_5 013.pdf.

[2] Coffey, A., \& Atkinson, P., (1996). Making sense of data qualitative data. Thousand Oaks: SAGE Publications.

[3] Cooper, D., \& Schindler, P. (2008). Business Research Methods. McGraw-Hill Companies, Inc. New York, NY.

[4] Creswell, J. W. (2007). Qualitative inquiry \& research design: Choosing among five approaches (2nd ed.). Thousand Oaks: SAGE Publications.

[5] Driscoll, C., \& McKee (2007). Restorying a culture of ethical and spiritual values: A role for leader storytelling. Journal of Business Ethics, 73 (pp. 205-217). http://dx.doi.org/10.1007/s10551-0069191-5

[6] Fontana, A. \& Frey, J. H. (2000). The interview: From structured questions to negotiated text. In Denzin, N. K., \& Lincoln, Y. S. The handbook of qualitative research (pp. 695-728). Thousand Oaks: SAGE Publications.

[7] Hart K. (2006, December/January) Inbox Journalism. American Journalism Review. Retrieved on January 13, 2012 from: http://www.ajr.org/article.asp?id=4005.

[8] Koestenbaum, P. (2002). The inner side of greatness: A philosophy for leaders. Jossey-Bass.

[9] Lambrecht, J. J. (1999). Developing Employment-Related Office Technology Skills (MDS-1199). Berkeley: National Center for Research in Vocational Education, University of California.

[10] Meho, L. I. (2006). E-mail interviewing in qualitative research: A methodologyical discussion. Journal of the American Society for Information Science and Technology, 57(10). http://dx.doi.org/ $\underline{10.1002 / a s i .20416}$

[11] Miles, M. B., \& Huberman, A. M. (1994). Qualitative data analysis. Thousand Oaks: SAGE Publications.

[12] Muldoon, S. D., \& Miller, S. (2005). Leadership: Interpreting life pattern and their managerial significance. Emerald Group Publishing Limited, 24(2).

[13] Patton, M. Q. (1990). Qualitative evaluation and research methods (2nd ed.). Newbury Park, CA: Sage.

[14] Stockes \& Bergin (2006). Methodology or “methodolatry"? An evaluation of focus groups and depth interview. Qualitative Market Research. Retrieved from ABI/INFORM Global on January 14, 2009.

[15] Swanson, R. A., \& Holton, E. F. (2005). Research in organizations: Foundations and methods of inquiry.San Francisco: BerretKoehler Publishers, Inc.

\section{AUTHORS}

Olubunmi Philip Aborisade, Ph.D. was with the State University of New York. He is now with the College of New Rochelle, New York, USA.

Received 06 June 17 2012. Published as resubmitted by the author 25 April 2013. 